\title{
Increase of major complications with a longer ischemia time in breast reconstruction with a free deep inferior epigastric perforator (DIEP)
} flap

\author{
Nadine S. Hillberg ${ }^{1}$ (D) - Jop Beugels ${ }^{1}$. Sander M. J. van Kuijk ${ }^{2} \cdot$ René R. J. W. van der Hulst $^{1} \cdot$ Stefania M. H. Tuinder $^{1}$
}

Received: 19 May 2019 / Accepted: 27 September 2019 /Published online: 8 November 2019

(C) The Author(s) 2019

\begin{abstract}
Background The deep inferior epigastric artery perforator (DIEP) flap is one of the most used free flaps for postmastectomy breast reconstruction. Prolonged ischemia can result in (partial) flap loss. The aim of this study was to evaluate the association between ischemia time and postoperative complications of DIEP flap breast reconstruction.

Methods A retrospective study of all patients who received a breast reconstructionwith aDIEP flap atMaastricht University Medical Center in theNetherlands, between January 2010 and June $2017(\mathrm{n}=677)$. The flaps were divided into two groups: flaps with an ischemia time less than $60 \mathrm{~min}$ and those with $60 \mathrm{~min}$ or more. Recipient site complications, in particular major complications equal to re-exploration, and partial or total flap loss were the primary outcome measures.

Results In 23.9\% of the 677 included DIEP flaps, the ischemia time was 60 min or longer. Within this group, a complication of the recipient site occurred in $30.9 \%$ of the flaps. A major complication occurred in $17.3 \%$ of the flaps with $60 \mathrm{~min}$ or more ischemia time. With regard to the flaps with less than 60-min ischemia time, a complication occurred in $22.1 \%$ of the cases of which $8.9 \%$ would be considered amajor complication. A significant association was found between ischemia time and major complications on univariate ( $p$ value $=0.003$ ) and multivariate analyses ( $\mathrm{p}$ value $=0.016$ )

Conclusions This study demonstrates that an ischemia time less than $60 \mathrm{~min}$ is associated with a significantly lower risk of major recipient site complications compared to an ischemia time of $60 \mathrm{~min}$ or more.

Level of evidence: Level III, therapeutic, risk/prognostic study.
\end{abstract}

Keywords free flap · deep inferior epigastric artery perforator flap · DIEP · DIEAP · Complications · ischemia time

\section{Introduction}

Utilization of the free skin flaps based on a single paraumbilical perforator vessel from the deep inferior epigastric artery was

Nadine S. Hillberg

n.hillberg@zuyderland.nl

1 Department of Plastic, Reconstructive \& Hand Surgery, Maastricht University Medical Center, POBox 5800, 6202 Maastricht, The Netherlands

2 Department of Clinical Epidemiology and Medical Technology Assessment, Maastricht University Medical Center, Maastricht, The Netherlands described by Koshima and Soeda, after which Allen and Treece introduced the DIEP flap for breast reconstruction in $1994[1,2]$. Nowadays, the deep inferior epigastric artery perforator (DIEP) flap is one of the most frequently used free flaps for autologous breast reconstruction.

Studies have shown that breast reconstruction with a DIEP flap is safe and has many advantages over other methods. But minor and major complications of the recipient site still occur. Several risk factors for complications have already been reported, including obesity, diabetes mellitus, radiation, and smoking [3-5]. However, substantial proof with regard to the influence of ischemia time remains relatively unknown.

During surgery, the blood flow of the free flap is interrupted for a period of time, also called the ischemia time. During this 
time, structural and metabolic changes can occur. These include the separation of leucocytes, the constriction of capillaries and metabolic dysfunction of endothelial cells [6]. These changes promote tissue injury by increased intracellular calcium concentration which can induce production and upregulation of inflammatory mediators. If the ischemia time is longer than the tissue's ischemia tolerance, tissue damage can occur $[7,8]$. Studies show that adipose tissue has a greater ischemia tolerance than skin tissue [9]. However, little has been reported with regard to the relation between ischemia time and the occurrence of complications and flap loss regarding breast reconstruction $[10,11]$.

The current hypothesis is that a longer ischemia time (i.e., over $60 \mathrm{~min}$ ) has an adverse effect on the survival of the flap. The aim of this study was to analyze the effect of $60 \mathrm{~min}$ or more ischemia on the development of complications and in particular major complications that lead to re-exploration and partial or total flap loss.

\section{Patients and methods}

A retrospective analysis of a prospectively collected cohort was performed on patients who underwent an immediate or delayed DIEP flap breast reconstruction at Maastricht University Medical Center (MUMC+) in the Netherlands between January 2010 and June 2017.

The study was performed in accordance with the ethical standards of the Declaration of Helsinki.

\section{Outcome measures}

Patient demographics and medical records were reviewed. Comorbidities, risk factors, oncological therapy, and operative details were collected. The postoperative follow-up period was at least 1 month. A complication was defined as any complication at the recipient site including wound problems, hematoma, seroma, fat necrosis, infection, re-exploration, and partial or total flap loss. Major complications were defined as partial or total flap loss or re-exploration.

Because we did not anticipate that there to be a linear association between ischemia time and the log-odds of experiencing a complication, but hypothesized that there would be a threshold after which ischemia would result in a higher likelihood of complications, we chose a cutoff value of $60 \mathrm{~min}$ based on consensus between the surgeons in our center involved in these procedures.

The ischemia time was collected from the operation report made by the plastic surgeon, in which the ischemia time is normally mentioned in minutes. If the time was not mentioned in the operation report, it was possible to get the data from the operation report of the anesthesiologist where the start and finish of the ischemia time is mentioned.

\section{Statistical analysis}

Descriptive statistics were used to summarize the demographic variables of the cohort. Mean and standard deviation (SD) were described for the continuous variables with a symmetric distribution and median with minimum and maximum ranges for continuous variables with a skewed distribution. Frequencies and percentages were used for categorical variables. We used stochastic regression imputation to impute missing values to prevent a loss of statistical power.

Demographic variables were compared between patients with an ischemia time less than $60 \mathrm{~min}(<60 \mathrm{~min})$ and an ischemia time $60 \mathrm{~min}$ or more ( $\geq 60 \mathrm{~min}$ ). Ischemia time was defined at the time between the interruption of blood supply of the flap and the restoration of sufficient circulation through the flap by a successful anastomosis of the pedicle. Differences in demographics between the ischemia groups were assessed using the Mann-Whitney $U$ test for continuous variables and chi-square tests, or Fischer's exact tests as appropriate for categorical variables. After an univariable analysis of the effect of ischemia time on risk of postoperative complications, a binary logistic regression analysis was used to assess the associations of clinically relevant factors including age at operation, body mass index (BMI), smoking status, weight of transplanted flap, ischemia time, radiotherapy, diabetes mellitus (DM), neoadjuvant chemotherapy, number of perforators, uni- or bilateral DIEP flap, and immediate or delayed reconstruction, with the occurrence of complications. The variables included in the logistic regression analysis were chosen based on literature and differences between groups [3, 4]. Due to a lack of events, we were not able to assess the associations of major complications with all the same factors in the logistic regression analysis. We decided to assess the associations of major complications with BMI, smoking status, weight of transplanted flap, ischemia time, radiotherapy, and chemotherapy. Some women underwent bilateral reconstruction and, hence, some observations were clustered within patients. Because of the low proportion of clustered data, we chose to use normal regression that does not account for uncorrelated data. As a sensitivity analysis, we repeated the analyses using generalized estimating equations (GEE) to assess whether models that account for clustering would provide different estimates.

A $p$ value $\leq 0.05$ was considered to be statistically significant. Data analyses were performed using SPSS software (version 23.0, SPSS INC, Chicago, IL, USA) for Windows [12].

\section{Results}

In this study, 677 deep inferior epigastric artery perforator (DIEP) flaps, in 471 patients, for breast reconstruction 
Table 1 Patient demographics of the different groups

\begin{tabular}{|c|c|c|c|c|}
\hline & Total & Ischemia time $<60 \mathrm{~min}$ & Ischemia time $\geq 60 \mathrm{~min}$ & $p$ value* \\
\hline DIEP flaps, $n$ & 677 & 515 & 162 & \\
\hline Age at operation, median (min-max), year & $49(20-81)$ & $49(24-81)$ & $50(20-72)$ & 0.071 \\
\hline Smoking, $n(\%)$ & $50(7.4 \%)$ & $35(6.8 \%)$ & $15(9.3 \%)$ & 0.296 \\
\hline Body mass index, mean (SD), $\mathrm{kg} / \mathrm{m}^{2}$ & $26.9(3.7)$ & $26.8(3.7)$ & $27.3(3.9)$ & 0.195 \\
\hline Diabetes mellitus, $n(\%)$ & $26(3.8 \%)$ & $22(4.3 \%)$ & $4(2.5 \%)$ & 0.298 \\
\hline Neo-adjuvant therapy, n (\%) & $135(19.9 \%)$ & $109(21.2 \%)$ & $26(16.0 \%)$ & 0.259 \\
\hline Immediate reconstruction, $n(\%)$ & $342(50.5 \%)$ & $268(52.0 \%)$ & $74(46.0 \%)$ & 0.158 \\
\hline Weight of transplanted flap, mean (SD), $100 \mathrm{~g}$ & $6.516(2.511)$ & $6.453(241.6)$ & $7.135(2.734)$ & $0.003 *$ \\
\hline Number of perforators per flap, $n(\%)$ & $485(71.6 \%)$ & $382(68.3 \%)$ & $103(63.6 \%)$ & $0.023^{*}$ \\
\hline 1 & $167(24.7 \%)$ & $114(22.1 \%)$ & $53(32.7 \%)$ & \\
\hline 2 & $25(3.7 \%)$ & $19(3.7 \%)$ & $6(3.7 \%)$ & \\
\hline \multicolumn{5}{|l|}{3 or more } \\
\hline Unilateral, $n(\%)$ & $235(34.7 \%)$ & $158(30.8 \%)$ & $77(47.5 \%)$ & $0.000^{*}$ \\
\hline Bilateral, $n(\%)$ & $412(60.9 \%)$ & $334(64.9 \%)$ & $78(48.1 \%)$ & \\
\hline Stacked unilateral, $n(\%)$ & $30(4.4 \%)$ & $23(4.5 \%)$ & $7(4.3 \%)$ & \\
\hline Adjuvant chemotherapy, $n(\%)$ & $245(36.2 \%)$ & $179(34.8 \%)$ & $66(40.7 \%)$ & 0.167 \\
\hline Radiation, $n(\%)$ & $189(27.9 \%)$ & $144(28.0 \%)$ & $45(27.8 \%)$ & 0.964 \\
\hline
\end{tabular}

* $p$ value between groups were assessed using the Mann-Whitney $U$ test for continuous variables and chi-square tests, or Fischer's exact tests as appropriate for categorical variables

$F$ assessed by Fischer's exact test

performed in Maastricht University Medical Center were included. Characteristics of all 677 flaps are summarized in Table 1.

The median age of the patients was 49 years. The mean BMI was $26.9 \mathrm{~kg} / \mathrm{m}^{2}$ (SD 3.7). Fifty (7.4\%) flaps were performed on patients who were active smokers at the time of the operation. Four hundred twelve (60.9\%) flaps were used for a bilateral DIEP reconstruction; 235 $(34.7 \%)$ were unilateral flaps, and $30(4.4 \%)$ flaps were stacked unilateral reconstructions. A total of 135 (19.9\%) of the flaps were performed on patients who had received neoadjuvant chemotherapy. The mean weight of the transplanted flap was $651.6 \mathrm{~g}$ (SD 251.6). In 485 $(71.6 \%)$ of the flaps, only one perforator was used for the anastomosis; in $167(24.7 \%)$ flaps, two perforators were used, and only in $25(3.7 \%)$ of the flaps, three or more perforators were re-attached. The median ischemia time was 51 (18-215) minutes per flap.

As shown in Table 1, the flaps were divided in two groups based on their ischemia time. One hundred sixtytwo $(23.9 \%)$ flaps were performed with an ischemia time $\geq 60 \mathrm{~min}$. Univariate analyses were performed in order to identify differences between groups. The weight of the transplanted flap was statistically significantly higher in the group with an ischemia time $\geq 60 \min$ ( $p$ value $=$ 0.003 ).

A comparison of complications between the groups categorized by ischemia time is shown in Table 2. In the group with an ischemia time of $\geq 60 \mathrm{~min}$, significantly, more complications occurred (30.9\%) compared to the flaps with an ischemia time of $<60 \mathrm{~min}(22.1 \%, p$ value $=0.024)$. Even when only major complications were taken into account, a statistically significant difference was observed between the two groups ( $p$ value $=0.003$ ).

Binary logistic regression analysis for overall complications was used to adjust for other factors including age at operation, diabetes mellitus, BMI, smoking status, weight of transplanted flap, ischemia time, radiotherapy, chemotherapy, number of perforators, uni- or bilateral DIEP flap, and immediate or delayed reconstruction. As shown in Table 3, the logistic regression analysis showed that the weight of the transplanted flap and BMI was significantly associated with postoperative complications (OR 1.110; $p=0.010$, OR 1.070; $p=$ $0.043)$. The other variables were not significantly associated with complications after a DIEP flap breast reconstruction.

Table 4 shows results from the binary logistic regression analysis for major complications adjusted for BMI, diabetes, weight of transplanted flap, ischemia time, radiotherapy, and neoadjuvant chemotherapy. The logistic regression analysis showed that the ischemia time was significantly associated with postoperative major complications (OR 1.891; $p=$ $0.016)$. In addition, a statistically significant association was found with regard to the weight of the transplanted flap (OR $1.110 ; p=0.015)$. 
Table 2 Complication rates between the groups

\begin{tabular}{llll}
\hline & Ischemia time $<60$ min & Ischemia time $\geq 60$ min & $p$ value* \\
\hline DIEP flaps, $n$ & 515 & 162 & \\
Wound problems, $n(\%)$ & $57(11.1 \%)$ & $18(11.1 \%)$ & 0.988 \\
Infection, $n(\%)$ & $32(6.2 \%)$ & $6(3.7 \%)$ & 0.226 \\
Hematoma, $n(\%)$ & $23(4.5 \%)$ & $12(7.4 \%)$ & 0.140 \\
Seroma, $n(\%)$ & $7(1.4 \%)$ & $2(1.2 \%)$ & $1.000^{\mathrm{F}}$ \\
Fat necrosis, $n(\%)$ & $38(7.4 \%)$ & $19(11.7 \%)$ & 0.082 \\
Re-exploration, $n(\%)$ & $34(6.6 \%)$ & $21(13.0 \%)$ & $0.010^{*}$ \\
Re-anastomosis, $n(\%)$ & $23(4.5 \%)$ & $7(4.3 \%)$ & 0.938 \\
Partial flap loss, $n(\%)$ & $17(3.3 \%)$ & $9(5.6 \%)$ & 0.193 \\
Total flap loss, $n(\%)$ & $10(1.9 \%)$ & $8(4.9 \%)$ & $0.050^{\mathrm{F}}$ \\
Overall complication, $n(\%)$ & $114(22.1 \%)$ & $50(30.9 \%)$ & $0.024 *$ \\
Major complication, $n(\%)$ & $46(8.9 \%)$ & $28(17.3 \%)$ & $0.003 *$ \\
\hline
\end{tabular}

* $p$ value between groups were assessed using the Mann-Whitney $U$ test for continuous variables and chi-square tests, or Fischer's exact tests as appropriate for categorical variables

$F$ assessed by Fischer's exact test

\section{Discussion}

The DIEP flap has become a frequently used method for autologous free flap breast reconstruction. In free flap operations, a period without blood supply to the flap is inevitable. Previous studies showed varying results when it comes to ischemia time and postoperative complications $[10,11$, 13-16]. Gurlek et al. showed that an ischemia time of less than $3 \mathrm{~h}$ in a free flap operation for breast or head and neck reconstruction did not affect the survival of the flap. However, according to a study by Lee et al., an ischemia time longer than 99.5 min significantly increases the risk of fat necrosis.

Table 3 Binary logistic regression analysis to assess the associations of overall complications

\begin{tabular}{lllll}
\hline & $p$ value & OR & \multicolumn{2}{l}{$95 \%$ CI } \\
\cline { 4 - 6 } & & & Lower & Upper \\
\hline Age at operation, year & 0.625 & 0.955 & 0.974 & 1.016 \\
Smoking & 0.160 & 1.597 & 0.831 & 3.069 \\
Body mass index, kg/m ${ }^{2}$ & 0.043 & 1.070 & 1.002 & 1.144 \\
Diabetes mellitus & 0.426 & 1.420 & 0.598 & 3.370 \\
Neo-adjuvant therapy & 0.246 & 0.722 & 0.417 & 1.251 \\
Immediate reconstruction & 0.724 & 0.930 & 0.622 & 1.391 \\
Weight of transplanted flap, $100 \mathrm{~g}$ & 0.010 & 1.110 & 1.000 & 1.221 \\
Number of perforators per flap & 0.694 & - & - & - \\
1 & 0.396 & 0.828 & 0.535 & 1.281 \\
2 & 0.838 & 0.907 & 0.354 & 2.320 \\
3 or more & & & & \\
Unilateral & 0.536 & - & - & - \\
Bilateral & & & & \\
Stacked unilateral & 0.267 & 0.786 & 0.514 & 1.202 \\
Radiation & 0.752 & 0.844 & 0.295 & 2.414 \\
Ischemia time, $<$ or $\geq 60$ min & 0.439 & 1.195 & 0.761 & 1.878 \\
\hline & 0.134 & 1.377 & 0.906 & 2.094 \\
\hline
\end{tabular}

But they do not report about other postoperative complications [10]. The study of Marre et al. reports a higher risk of vascular compromise in DIEP flaps for breast reconstruction with an ischemia time longer than $2 \mathrm{~h}$ [11]. In our study, an increased risk of major postoperative complications was identified in DIEP flaps with an ischemia time $\geq 60 \mathrm{~min}$. The cutoff point of $60 \mathrm{~min}$ of ischemia time was chosen based on the rounded up average ischemia time of the first five and last five DIEP-flaps (53 min) without any major postoperative complications. Furthermore, an anastomosis should usually be achievable within $60 \mathrm{~min}$, with sufficient time to give a resident the opportunity to learn the procedure.

This study shows that a longer ischemia time in DIEP flap for breast reconstruction is statistically significantly associated with a higher risk of major postoperative complications of the recipient site. An ischemia time of $\geq 60 \mathrm{~min}$ increases the chance of re-exploration or (partial) flap loss. A short ischemia time can be achieved by ensuring that the OR-team is wellinstructed, familiar with the procedure and that all equipment is ready before the ischemia time starts. A good balance has to

Table 4 Binary logistic regression analysis to assess the associations of major complications

\begin{tabular}{lccccc}
\hline & $p$ value & OR & \multicolumn{2}{l}{$95 \% \mathrm{CI}$} \\
\cline { 4 - 6 } & & & Lower & Upper \\
\hline Body mass index, $\mathrm{kg} / \mathrm{m}^{2}$ & 0.701 & 0.984 & 0.906 & 1.069 \\
Smoking & 0.582 & 1.272 & 0.541 & 2.990 \\
Neo-adjuvant therapy & 0.247 & 0.653 & 0.318 & 1.343 \\
Weight of transplanted flap, $100 \mathrm{~g}$ & 0.015 & 1.110 & 1.000 & 1.350 \\
Radiation & 0.412 & 0.780 & 0.431 & 1.412 \\
Ischemia time, $<$ or $\geq 60 \mathrm{~min}$ & 0.016 & 1.891 & 1.126 & 3.177 \\
\hline
\end{tabular}


be made between making the anastomosis by experienced micro-surgeons and the residents.

A bias of the study could be that a longer ischemia can also be caused by a more complicated anastomosis for several reasons. For example, a bad condition of the vessels may complicate the anastomosis and prolong the ischemia time. Bad condition of vessels themselves could also, independently of the ischemia time, increase the risk of postoperative flap complications. However, also in cases in which ischemia time might be a reflection of a difficult technical procedure, it remains a predictive indicator for the risk of major complications postoperatively.

We observed differences in flap weight between the groups based on ischemia time $(p=0.003)$. This study also demonstrates that the weight of the transplanted flap showed a significantrelation to both overall and major complications (OR 1.110; $p=0.010)$. Therefore, flap weight was corrected for in the multivariable logistic regression analysis when analyzing the correlation of ischemia time and postoperative complications. Flaps can be heavier because they are thicker or because they have a larger surface area. It might be worth investigating whether the risk of postoperative complications with these flaps can be reduced by making heavier flaps bipedicled to create a more secure blood flow.

As shown in Table 3, a statistically significant association was found between overall complications and BMI $(p=0.043)$. This corresponds with previous studies that conclude that there is a higher risk of complications in patients with a higher BMI $[3,9]$. No association was found for major complications and BMI $(p=0.701)$. Tissue damage in free flap operations can occur due to various causes. Inadequate perfusion of the flap, because of insufficient connection between the smaller vessels and the connected perforator is an important cause of tissue damage. However, tissue damage can also occur due to an ischemia time longer than the tissue's ischemia tolerance. The forgoing causes irreversible damage to the tissue that cannot recover sufficiently after regaining sufficient blood flow through the anastomosis. Clinically, distinguishing between the two different causes of tissue damage is very difficult. More often, an ICG-camera is used to assess the blood flow in a flap. The ICG-camera can also be used in free flap operations but does not distinguish the cause of the reduced blood flow $[17,18]$. It would be interesting to develop a method to investigate and distinguish the various causes of tissue damage.

The causes of tissue damage have to be studied in relation to the difference in tissue ischemia time. For example, it is quite conceivable that a free flap harvested from the gluteal area has a greater tissue ischemia tolerance compared to the DIEP flap, since the tissue of this area is exposed to a reduced blood flow more often due to prolonged pressure on the tissue by sitting. To know whether tissue that is more often intermittently exposed to ischemia also has a higher tissue ischemia tolerance could be of great value for free flap reconstructions.

This study is unique due to the large number of similar types of free flap breast reconstructions, all performed in one hospital and by a small number of plastic surgeons all using the same technique. The study therefore concerns a unique population with strong evidence that ischemia time is important. Translating these results into clinical practice strongly suggests that when the resident is making the anastomosis and it still goes very slowly at an ischemia time of $40 \mathrm{~min}$, it may be wise to let a staff member finish the anastomosis to keep the ischemia time $<60 \mathrm{~min}$. Or when intraoperatively, the vessels seem of such a bad condition that it is expected that the anastomosis is likely to become very difficult, it may be a good decision to use other vessels for the anastomosis to decrease the ischemia time of the flap and the risk of postoperative complications.

Heavier flaps seem to have a higher risk of postoperative complications; for those flaps, it may be even more important to stick to an ischemia time $<60 \mathrm{~min}$. It would be interesting to explore if reconstruction of bigger breast by bipedicled flaps could reduce the risk of postoperative complications compared to reconstructions with unipedicled flaps.

\section{Conclusion}

This study shows that it is useful to aim for an intraoperative ischemia time of less than 60 min when performing a DIEP flap for reconstruction of the breast. With a longer ischemia time, there is a greater risk of major complications at the recipient site postoperatively.

\section{Compliance with ethical standards}

Ethical StatementConflict of interest The authors declare that they have no conflict of interest.

Ethical approval The study consisted of a chart study. All procedures performed in this study involving human participants were in accordance with the 1964 Helsinki declaration and its later amendments or comparable ethical standards.

Informed consent The study consisted of an anonymous retrospective chart study. No individual informed consent of the patients was needed for this study.

Open Access This article is distributed under the terms of the Creative Commons Attribution 4.0 International License (http:// creativecommons.org/licenses/by/4.0/), which permits unrestricted use, distribution, and reproduction in any medium, provided you give appropriate credit to the original author(s) and the source, provide a link to the Creative Commons license, and indicate if changes were made.

\section{References}

1. Koshima I, Soeda S (1989) Inferior epigastric artery skin flaps without rectus abdominis muscle. Br J Plast Surg 42(6):645-648 
2. Allen RJ, Treece P (1994) Deep inferior epigastric perforator flap for breast reconstruction. Ann Plast Surg 32(1):32-38

3. Beugels J, Hoekstra LT, Tuinder SM, Heuts EM, van der Hulst RR, Piatkowski AA (2016) Complications in unilateral versus bilateral deep inferior epigastric artery perforator flap breast reconstructions: a multicentre study. J Plast Reconstr Aesthet Surg 69(9):1291-1298

4. Wang XL, Liu LB, Song FM, Wang QY (2014) Meta-analysis of the safety and factors contributing to complications of MS-TRAM, DIEP, and SIEA flaps for breast reconstruction. Aesthet Plast Surg 38(4):681-691

5. Voineskos SH, Frank SG, Cordeiro PG (2015) Breast reconstruction following conservative mastectomies: predictors of complications and outcomes. Gland Surg 4(6):484-496

6. Siemionow M, Arslan E (2004) Ischemic/reperfusion injury: a review in relation to free tissue transfers. Microsurgery. 24(6):468475

7. Widgerow $A D$ (2014) Ischemic-reperfusion injury: influencing the microcirculatory and cellular environment. Ann Plast Surg 72(2): 253-260

8. Morris SF, Pang CY, Zhong A, Boyd B, Forrest CR (1993) Assessment of ischemic-induced reperfusion injury in the pig latissimus dorsi myocutaneous flap model. Plast Reconstr Surg 92(6):1162-1172

9. Chang DW, Wang B, Robb GL et al (2000) Effect of obesity on flap and donor-site complications in free transverse rectus abdominis myocutaneous flap breast reconstruction. Plast Reconstr Surg 105(5):1640-1648

10. Lee KT, Lee JE, Nam SJ, Mun GH (2013) Ischemic time and fat necrosis in breast reconstruction with a free deep inferior epigastric perforator flap. J Plast Reconstr Aesthet Surg 66(2):174-181
11. Marre D, Hontanilla B (2013) Increments in ischaemia time induces microvascular complications in the DIEP flap for breast reconstruction. J Plast Reconstr Aesthet Surg 66(1):80-86

12. IBM Corp. Released 2015. IBM SPSS Statistics for Windows, Version 23.0. Armonk, NY: IBM Corp.

13. Ochoa O, Chrysopoulo M, Nastala C, Ledoux P, Pisano S (2012) Abdominal wall stability and flap complications after deep inferior epigastric perforator flap breast reconstruction: does body mass index make a difference? Analysis of 418 patients and 639 flaps. Plast Reconstr Surg 130(1):21e-33e

14. Damen TH, Morritt AN, Zhong T, Ahmad J, Hofer SO (2013) Improving outcomes in microsurgical breast reconstruction: lessons learnt from 406 consecutive DIEP/TRAM flaps performed by a single surgeon. J Plast Reconstr Aesthet Surg 66(8):1032-1038

15. van den Heuvel MG, Buurman WA, Bast A, van der Hulst RR (2009) Review: ischaemia-reperfusion injury in flap surgery. J Plast Reconstr Aesthet Surg 62(6):721-726

16. Gurlek A, Kroll SS, Schusterman MA (1997) Ischemic time and free flap success. Ann Plast Surg 38(5):503-505

17. Malagon-Lopez P, Vila J, Carrasco-Lopez C et al (2018) Intraoperative indocyanine green angiography for fat necrosis reduction in the deep inferior epigastric perforator (DIEP) flap. Aesthet Surg J

18. Bigdeli AK, Gazyakan E, Schmidt VJ et al (2016) Indocyanine green fluorescence for free-flap perfusion imaging revisited: advanced decision making by virtual perfusion reality in visionsense fusion imaging angiography. Surg Innov 23(3):249-260

Publisher's note Springer Nature remains neutral with regard to jurisdictional claims in published maps and institutional affiliations. 